\title{
Financement de la planification familiale
}

\author{
Moazzam Ali \\ Benjamin Bellows \\ Population Council
}

Follow this and additional works at: https://knowledgecommons.popcouncil.org/departments_sbsr-rh

Part of the Social and Behavioral Sciences Commons How does access to this work benefit you? Let us know!

\section{Recommended Citation}

Ali, Moazzam and Benjamin Bellows. 2018. "Financement de la planification familiale," Résumés des preuves sur la planification familiale. New York: Population Council. 


\section{Financement de la planification familiale}

\section{$\rightarrow$ Assurer le financement approprié des produits et des services de planification familiale}

L'augmentation efficace et efficiente des investissements dans la planification familiale par les secteurs public et privé est essentiel pour atteindre l'objectif du programme PF2020 qui vise à aider 120 millions de femmes supplémentaires à devenir des utilisatrices de la contraception moderne. Malgré les efforts déployés par les gouvernements nationaux, les donateurs et les particuliers sont responsables de près de la moitié ( $49 \%$ ) des coûts de la santé reproductive, maternelle, néonatale et des enfants (1). Les dépenses des ménages éclipsent les contributions des sources de financement nationales et internationales. Les efforts futurs visant à réduire les besoins non satisfaits en matière de PF doivent tenir compte des coûts payés par les consommateurs, de leur rentabilité et des sources de financement.

\section{DÉFIS AU FINANCEMENT DE LA PLANIFICATION FAMILIALE}

Le coût annuel direct et indirect estimatif de la fourniture de services de contraception modernes à 671 millions d'utilisateurs dans les régions en développement est de 6,3 milliards USD (2). Les coûts directs comprennent les contraceptifs, les fournitures et les salaires des travailleurs de la santé. Les coûts indirects comprennent l'appui aux programmes, l'information et l'éducation sur la PF, la construction et l'entretien des établissements, ainsi que la gestion de la chaîne d'approvisionnement. II en résulte un coût moyen (y compris direct et indirect) de 1,01 USD pour la contraception moderne par personne et par an dans les régions en développement.

L'élargissement et l'amélioration des services pour répondre aux besoins de toutes les femmes et filles dans les régions en développement (214 millions supplémentaires) pour la contraception moderne coûteraient 12 milliards USD par an ( $y$ compris direct et indirect) ou 1,93 USD par personne et par an (2).

Le coût moyen par personne dans les régions en développement pour les services de contraception moderne et les soins maternels et néonatals s'élève à 8,56 USD ou 53,6 milliards USD par an (2). L'investissement dans les services contraceptifs et les services de santé maternelle et néonatale se traduit par une économie nette de 6,9 milliards USD par rapport à l'investissement dans les soins de santé maternelle et néonatale.

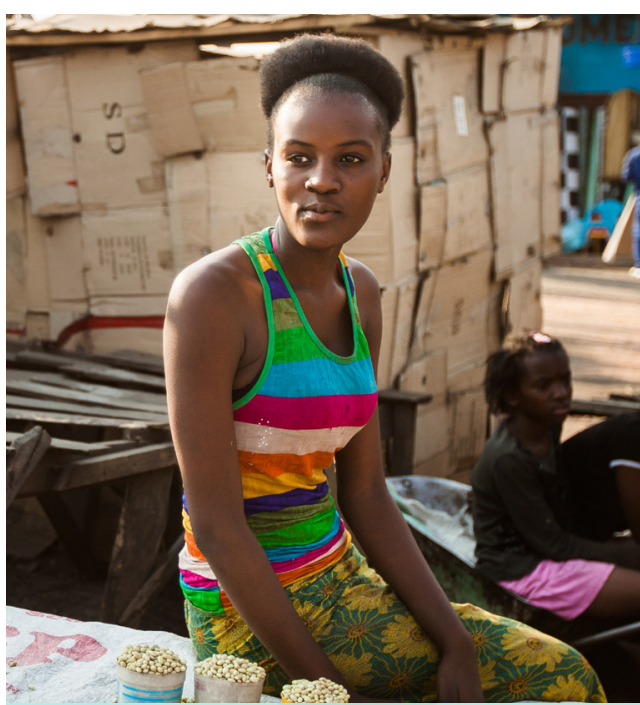

\section{CONSIDÉRATIONS POLITIQUES ET PROGRAMMATIQUES}

$\rightarrow$ Assurer une collecte de fonds, une mise en commun et des achats stratégiques durables afin d'intensifier les systèmes de prestation des méthodes contraceptives.

$\rightarrow$ Augmenter le nombre d'utilisateurs de la planification familiale supplémentaires en atteignant de nouveaux utilisateurs et en améliorant les taux de continuation parmi les utilisateurs actuels.

$\rightarrow$ Effectuer des recherches plus poussées sur les mesures de la qualité de la planification familiale, comme l'information sur les méthodes pour tester leur corrélation avec des taux de continuation plus élevés. L'insatisfaction des clients et l'abandon représentent des risques significatifs pour le succès du programme PF2O2O. 
Si les tendances actuelles de l'utilisation des contraceptifs dans 148 pays en développement et territoires se poursuivent, il y aura un déficit de financement de 322 millions USD en 2020 pour les seuls produits de base (2). Si l'objectif du programme PF2020 était pleinement atteint, l'écart de financement restant entre les montants dépensés pour les fournitures en 2014 et 2020 serait de 541 millions USD (3).

Les ressources des donateurs ont augmenté au fil du temps, les États-Unis étant historiquement le plus grand donateur bilatéral suivi par le Royaume-Uni (3). En 2015, les États-Unis ont contribué à près de la moitié (47\%) du financement bilatéral pour la PF, soit 638 millions USD et le Royaume-Uni a contribué à hauteur de 269,9 millions USD $(20 \%)(4)$.

Il existe également des sources de financement multilatérales, le FNUAP dépensant environ $43 \%$ de ses fonds, soit 341 millions USD en faveur de la PF en 2015, et la Banque mondiale, 251 millions USD en matière de population et de santé procréative. (4). À la suite de la création de la Facilité de financement mondiale (FFM), la Banque mondiale devrait jouer un rôle plus important dans la PF (4).

Les fondations et le secteur privé ont également apporté des contributions importantes à la santé reproductive, la Fondation Bill \& Melinda Gates, par exemple, consacrant 148 millions USD à la planification familiale en 2015 (4). Et les entreprises pharmaceutiques ont joué un rôle en partenariat avec les donateurs grâce à des mécanismes de financement novateurs qui ont vu des réductions significatives du prix des produits contraceptifs - notamment par Bayer et Merck pour les implants contraceptifs.

Toutefois, les perspectives de financement futur des donateurs sont incertaines compte tenu de l'instabilité des taux de change, de l'évolution des programmes politiques des pays donateurs, de la création de la FFM et des autres évolutions mondiales. Le budget américain de 2018 a retenu sa contribution au FNUAP et laissé le financement pour la planification familiale inchangé; néanmoins, des réductions importantes sont prévues en 2019 (4).

En outre, les engagements de financement nationaux sont très variables d'un pays à l'autre. Le financement intérieur exigera probablement davantage d'engagement des ministères des finances et des ministères de la Santé pour mettre l'accent sur le dividende démographique. Le dividende démographique est une augmentation de la croissance et du développement économiques qui pourraient être atteints si l'évolution de la structure de l'âge de la population entraîne une baisse des taux de fécondité à la suite d'une utilisation accrue de la contraception associée à des investissements pour améliorer l'éducation, la création d'emplois, l'efficacité de la production de recettes et de la perception des impôts et l'augmentation des taux d'imposition (1). Une plus grande intégration des secteurs public et privé dans la prestation des services, la mise en commun des achats et l'achat stratégique aboutiront à des dépenses plus efficaces et plus élevées par habitant grâce à des investissements plus conséquents. Le fait de servir les femmes et les populations difficiles à atteindre dans les pays à faible revenu exige une approche intégrée du renforcement des systèmes de santé afin de progresser vers une couverture sanitaire universelle (1).

\section{CONSIDÉRATIONS POUR ASSURER LE FINANCEMENT APPROPRIÉ DES PRODUITS ET DES SERVICES DE PLANIFICATION FAMILIALES}

Augmentation du nombre d'utilisateurs de la planification familiale supplémentaires en atteignant de nouveaux utilisateurs et en améliorant les taux de continuation des utilisateurs actuels $(5,6)$. Des recherches supplémentaires sur les paramètres de performance de la qualité de la PF, comme l'indice d'information sur la méthode, sont nécessaires pour tester leur corrélation avec des taux de continuation améliorés. En particulier, à mesure que la prévalence de la contraception augmente, l'insatisfaction et l'abandon des clients représentent un risque important pour le succès de l'initiative PF2O2O (7).

L'accès universel à la planification familiale serait l'un des rapports avantages-coûts les plus élevés parmi un large choix d'options politiques pour le développement (8). Les avantages sociaux et économiques pour les femmes, leurs familles et les sociétés découleront de l'augmentation de l'éducation des femmes et des enfants, de l'augmentation des revenus des femmes et de nouvelles réductions de la pauvreté (2). En matière de santé, la prévention est beaucoup moins onéreuse que le traitement ; le coût de la contraception moderne est beaucoup plus faible que la prestation de soins pour les grossesses non désirées. Pour chaque dollar supplémentaire dépensé en PF au-dessus du niveau actuel, le coût des soins liés à la grossesse dans les régions en développement est réduit de 2,20 USD (2). $\mathrm{Si}$ les besoins en matière de contraception moderne et de soins aux mères et aux nouveau-nés étaient pleinement satisfaits, le résultat serait une économie nette de 6,9 milliards USD par rapport à l'investissement dans les soins de santé maternelle et néonatale (2). L'investissement dans la contraception moderne procurera un retour sur investissement important qui augmentera avec le temps (12). 


\section{RÉFÉRENCES}

1 Lie, G.S., A.L. Soucat, et S. Basu. 2015. "Financing women's, children's, and adolescents' health," British Medical Journal 14 sept., 351: h4267.

2 Guttmacher Institute. 2017. Adding It Up: Investing in Contraception and Maternal and Newborn Health, 2017. New York: Guttmacher Institute, 6 décembre.

3 Reproductive Health Supplies Coalition. 2016. "Global Contraceptive Commodity Gap Analysis 2016." Brussels: Reproductive Health Supplies Coalition. https://www. rhsupplies.org/uploads/tx_rhscpublications/Global_ Contraceptive_Commodity_Gap_Analysis_2016.pdf accédé le 14 juin, 2017.

4 The U.S. Government and International Family Planning \& Reproductive Health Efforts https://www.kff.org/ global-health-policy/fact-sheet/the-u-s-governmentand-international-family-planning-reproductive-healthefforts/

5 Kaiser Family Foundation. 2017. "Family Planning \& Reproductive Health Funding." Menlo Park, CA: Kaiser Family Foundation. http://www.kff.org/interactive/ budget-tracker/summary/Filter-Program-Area/FamilyPlanning_Reproductive-Health/Agency/?view=compareyears\&startYear=2017\&endYear=2018 (Accédé le 14 juin, 2017)

6 Dasgupta, A., M. Weinberger, B. Bellows, et W. Brown. 2017. " 'New users'are confusing our counting: Reaching consensus on how to measure 'additional users' of family planning," Global Health: Science and Practice 5(1): 6-14. Accédé à http://www.ghspjournal.org/ content/5/1/6.full.html

7 Jain, A.K., F. Obare, S. RamaRao, et I. Askew. 2013. "Reducing unmet need by supporting women with met need," International Perspectives on Sexual and Reproductive Health 39(3): 133-141. https://doi. org/10.1363/3913313

8 Castle, S. et I. Askew. 2015. Contraceptive Discontinuation: Reasons, Challenges and Solutions. New York: Population Council. Accédé à http://www. familyplanning2020.org/microsite/contraceptivediscontinuation

9 Kohler, H. et J.R. Behrman. 2014. Population and Demography Assessment Paper: Benefits and Costs of the Population and Demography Targets for the Post-2015 Development Agenda. Tewksbury, MA et Philadelphie: Copenhagen Consensus Center, University of Pennsylvania.
Auteurs : Moazzam Ali (Organisation mondiale de la Santé), Ben Bellows (Population Council)

Ce document fait partie de l'un des sept Résumés des preuves sur la planification familiale préparés dans le cadre du Sommet de la planification familiale tenu à Londres le 11 juillet 2017. Les résumés mettent en exergue les éléments de preuve et fournissent des considérations sur la recherche et les programmes pour améliorer l'accès à la planification familiale et réduire les grossesses non désirées. Les considérations relatives aux programmes sont basées sur les opinions expertes des auteurs, qui ont procédé à des examens documentaires sur la base des preuves existantes.

\section{Résumés des preuves sur la planification familiale}

- Accélérer l'adoption de la planification familiale volontaire et fondée sur les droits dans les pays en développement (vue d'ensemble) (mise à jour octobre 2018)

- Financement de la planification familiale (mise à jour octobre 2018)

- Réduction des grossesses précoces et involontaires chez les adolescentes (mise à jour octobre 2018)

- Amélioration de la prestation de services de planification familiale pendant les crises humanitaires

- Assurer la sécurité des contraceptifs par chaînes d'approvisionnement efficaces

- Élargissement du choix des contraceptifs (mise à jour octobre 2018)

- Partenariat avec le secteur privé pour renforcer la fourniture de la contraception

Seuls les auteurs sont responsables des opinions exprimées dans cet article qui ne représentent pas nécessairement les opinions, décisions ou politiques des institutions avec lesquelles ils sont affiliés.

La présente traduction n'a pas été établie par l'Organisation mondiale de la Santé (OMS). L'OMS ne saurait être tenue pour responsable du contenu ou de l'exactitude de la présente traduction. L'édition originale anglaise est l'édition authentique qui fait foi.

Family Planning Evidence Brief-Accelerating uptake of family planning: WHO/RHR/17.07

Family Planning Evidence Brief-Partnering with the private sector: WHO/ RHR/17.08

Family Planning Evidence Brief-Ensuring contraceptive security through effective supply chains: WHO/RHR/17.09

Family Planning Evidence Brief-Reducing early and unintended pregnancies among adolescents: WHO/RHR/17.10

Family Planning Evidence Brief-Reducing early and unintended pregnancies among adolescents: programme priorities: WHO/RHR/17.11 Family Planning Evidence Brief-Ensuring adequate financing of family planning commodities and services: WHO/RHR/17.12

Family Planning Evidence Brief-Improving family planning service delivery in humanitarian crises: WHO/RHR/17.13

Family Planning Evidence Brief-Expanding contraceptive choice: WHO/ $\mathrm{RHR} / 17.14$

Ce document a été financé par UK AID du gouvernement britannique ; toutefois, les opinions exprimées ne reflètent pas nécessairement les politiques du gouvernement britannique.

Préparé en juillet 2017. Mis à jour octobre 2018 